einander zeigen, und die, soviel ich weiss, nur mit Hülfe eines Cometensuchers bestimmt wurden. *) - Die Bahn dieses Cometen scheint somit zu den sicher elliptischen zu gehören, und die Unlaufszeit möchte wohl auf einige Jahre zu verhürgen sein. Auf die merkliche Abweichung der Bahn von der Parabel war bekanntlich Herr JumesWatson in Nö106 des Astronom. Journal zuerst aufmerksam geworden. Kopenhagen 1858 März 10.

Hans Lind.

\section{Schreiben des Herrn Observatory of Harvard College. Cambridge U.S.A. 1858 Febr. 15.}

Your notice of the Comet IV. of 1857 in the Astr. Nachr. ME 1123 induces me to take the liberty of forwarding to you by mail the two latest determinations of its position, made at this observatory. The observation of 0 ct. $21 / 22$ was made with difficulty, not so much from the faintness of the comet as from a slight haze in the sky. There were 4 passages in Right Ascension and 1 observation in Decl., which could not be repeated on account of the approach of day-light. There was however a very small star $\left(15^{\text {th }} \mathrm{mag}\right.$.) close to its centre and $I$ have recently verified the positions obtained by reexamining the region. The small star was at once recognised and on comparing its place with a star of the 8/9th mag. No 63 of Arg. Zones, the place given of the comet was fully confirmed. I have been thus particular with this observation as it will be very important in determining the orbit, being six weeks later than the European positions, which you have employed. an Herrn Observator Pape.

$$
\text { Comet IV. } 1857 .
$$

1857 Sept. $2316^{\text {h }} 21^{\text {m }} 45^{\text {' }} 5$ Cambr. m.s.t. Coniet's apparent AR $8^{\text {h }} 39^{\mathrm{n}} 55^{\mathrm{N}} 50$

$$
=\quad=\text { Decl. }-3^{\circ} 59^{\prime} 00^{\prime \prime} 5
$$

Star of Comp. W. vill. 1071.

Octob. $2117^{\text {h }} 6^{\mathrm{m}} 4^{\mathrm{s}} 0$ Cambr. n. s. t. AR $9^{\mathrm{b}} 34^{\mathrm{m}} 28^{\mathrm{b}} 32$

Decl. $-16^{\circ} 23^{\prime} 7^{\prime \prime} 1$

Star of Comp. $11^{\text {th }} \mathrm{mag}$. $=a$

$$
\begin{aligned}
& 8 / 9==b=\text { Arg. Z. Jo } 63 \\
& a \text { south of } b \quad 3^{\prime} 00^{\prime \prime} 7 \\
& a \text { precedes } b \quad 3^{\pi /} 39^{*} 05
\end{aligned}
$$

Comet north of $a 1^{\prime} 28^{\prime \prime} 1 \quad 1$ obs. at $7^{\mathrm{hh}} 4^{\mathrm{m}} 00^{\mathrm{s}}$ sid.time

$$
=\text { follows } a 0^{\mathrm{m} 5} 5^{8} 654=\frac{7910}{\text { Interval } 510}=
$$

Corr. for motion in Decl. in $5^{\mathrm{m}} 10-4^{\prime \prime} 5$

No correction has been applied for Refr. or Parallax.

\title{
Beobachtung des Laurent'schen Planeten zu Bilk.
}

1858 mittl. Zt. Bilk (51) zehnter Grösse.

März $5 \quad 8^{\text {h }} 56^{\prime \prime} 40^{\prime} 3 \quad 176^{\circ} 9^{\prime} 22^{\prime \prime} 3+0^{\circ} 10^{\prime} 19^{\prime \prime} 2 \quad 6$ Vergl.

Scheinb. Ort des Vergleichsterns nach Bessel Z. 75

(8.9) $176^{\circ} 56^{\prime} 40^{\prime \prime} 1+0^{\circ} 13^{\prime} 46^{\prime \prime} 0$

Tägliche Bewegung (51) $-44^{s}+11^{\prime}$.
Den Planeten (62, welcher, wie Herr Goldschmidt mir schrieb, durch den Herrn Marechal Vaillant „Europa“ benannt wurde, habe ich schon mehrmals beobachtet, aber die Beobachtungen der Vergl.-Sterne wegen noch nicht reducirt. Der Goldschnidt'sche Planet (48) heisst "Doris".

\section{R. Luther.}

\section{Entdeckung eines Cometen.}

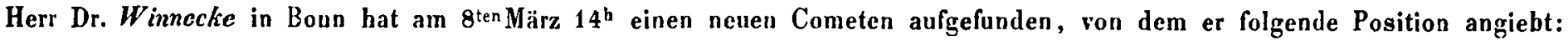

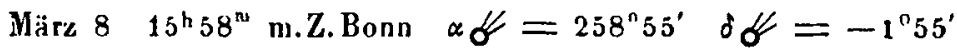

Tägliche Bewegung $\Delta \alpha=+1^{\circ} 8, \Delta \delta=0^{\circ} 0$

Herr Dr. Winnecke fügt hinzu: Der Comet ist gegen drei Minuten gross und verwaschen.

$\boldsymbol{P}$.

*) Diese Beobb. wurden aus $N 1107$ der Astr. Nachr. genommen; in . 10105 des Atr. Journ. otehen sie ein wenig davon abweichend. 